\title{
CAN POLYMORPHISM BE MAINTAINED BY SELECTION FAVOURING AN INTERMEDIATE OPTIMUM PHENOTYPE?
}

\author{
ROBERT SEMEONOFF \\ Department of Genetics, University of Leicester LEI 7RH, England
}

Received 6.xii.76

\begin{abstract}
Summary
Populations of Drosophila melanogaster containing the special chromosome, M-5, were subjected to artificial selection to canalise scutellar bristle phenotype at four bristles. At the end of the experiment allele frequencies at three enzyme loci were measured. In the selected population, these frequencies were found to have diverged from their starting values less than would have been predicted on the basis of random genetic drift. This was not found in control populations or in populations selected for low bristle number.
\end{abstract}

\section{INTRODUGTION}

Although the high degree of genetic variability in natural populations has been accepted for at least 10 years, the "selectionist" versus " neutralist" arguments to explain it continue.

Lewontin (1974) has suggested that a study of individual loci or groups of loci is unlikely to resolve the problem and that the influence of selection on the whole genome must be investigated. In particular, he claims that selection which favours intermediate phenotypes within a range will tend to establish long-lived quasi-equilibria in which segments of chromosome, considered in terms of the alleles they carry, will exist as a rather small number of complementary pairs (Lewontin, 1964). Many loci will be found to be polymorphic, and linkage disequilibrium between them will be large. Single locus polymorphisms then become windows into the chromosome through which we see evidence for what is ultimately polymorphisms for chromosome segments.

An experiment which appeared to demonstrate the operation of balanced selection on a locus was that of Yarbrough and Kojima (1967). This was a perturbation experiment in which populations were established at atypical allele frequencies for the Est-6 locus of Drosophila melanogaster. An equilibrium frequency was attained and was claimed to represent an optimum value for the population.

Criticism of this experiment (Yamazaki, 1971) suggested that what was being demonstrated was selection not at the Est-6 locus itself, but at any number of loci in an associated block about 5 map units long. Yarbrough and Kojima's lines were ultimately derived from a cross between two inbred lines. In spite of the 50 or so generations which intervened before the start of the experiment, some residual linkage disequilibrium would have persisted. In Lewontin's terms, what happened was that selection produced "classes of repulsion gametes" in which many loci were being held polymorphic. One of these was Est-6. Jones and Yamazaki (1974) report similar results in which the initial linkage disequilibrium was very much greater. Here, two lines homozygous for the appropriate alleles were used without 
the initial random breeding carried out by Yarbrough and Kojima. Jones and Yamazaki went on to show that when a large number of completely indepcndent lines were used to start the population, allele frequencies remained approximately constant.

Once we abandon claims to be able to detect selection at single loci and accept that some degree of linkage disequilibrium is almost unavoidable in artificial populations, it becomes less important to ensure a uniform genetic background for our chosen polymorphic loci. A founder population with a known high value for linkage disequilibrium will, in the absencc of selection, progress in a predictable way to equilibrium, and if it is kept small, loci which were originally polymorphic will similarly progress to fixation. If imposed selection favours some intermediate phenotype, both these processes may possibly be delayed and the genetic variability of the population preserved.

With this in mind I established a founder population of Drosophila melanogaster, subjected it to artificial selection for an intermediate phenotypc, that is to say, stabilising selection, and examined the allele frequencies at three polymorphic loci. It was not possible to examine linkage disequilibrium itsclf.

The character used was scutcllar bristle number in a population carrying the special X chromosome Muller-5 (In (1) $\mathrm{sc}^{\mathrm{Sil}} \mathrm{Lc}^{8 \mathrm{R}}+\mathrm{S}, \mathrm{sc}^{\mathrm{S1}} \mathrm{sc}^{8} \mathrm{w}^{\mathrm{a}} \mathrm{B}$ ), also known as M-5 or Basc. Under the conditions of my experiment, the effect of the two scute alleles is to disrupt the normal pattern of four scutellar bristles in hemizygous males. Most abnormal flies have less than four bristles, but a few may have five, or even six. The selection regime favoured flies with exactly four bristles. One reason for expecting a response to selection came from a casual observation made while maintaining stocks in which the M-5 chromosome was used to balance sex-linked lethals produced by X-irridation. When ncwly created, such stocks showed a wide distribution of scutellar bristle number in males, but after a year almost all males had four bristles. This was presumably due to the operation of natural selection. Also, Rendel and Sheldon (1960) using a different scute mutation showed that selection could reduce the variability of bristle number.

If variability is preserved in lines subjected to natural selection, the variance of gene frequencies after a number of generations will remain smaller than the value predicted by a model involving random drift alone. It is possible that the enzyme loci themselves, or others closely linked to them, might be subject to some independent form of stabilising selection. The properties of the M-5 chromosome allowed me to establish control lines in which wild-type males would be used as fathers. These are the $\mathrm{C}$ lines described below. Provided such independent selection is absent, the changes in gene frequencies in these lines should conform closely to the random drift model.

My results seem to show that the progress of polymorphic loci towards fixation can be retarded by artificial stabilising selection.

\section{Materials and methods}

(i) Stocks

An inbred wild-type stock and a stock containing the M-5 chromosome were obtained from the University of Birmingham, England. These stocks 
were found to differ at the loci Est-6 (3-36-8), Aph (3-46-3) and Adh (2-50-1). The wild-type stock was homozygous for the fast allele and the M-5 stock for the slow allele in each case.

The founder population consisted of the $\mathrm{F}_{2}$ generation from a cross between these two stocks. The distribution of scutellar bristles in 253 flies was:

$\begin{array}{llrrrr}\text { Number of bristles } & 1 & 2 & 3 & 4 & 5 \\ \text { Number of flies } & 8 & 20 & 77 & 140 & 8\end{array}$

The mean bristle count was 3.47

(ii) Selection lines

Four sets of three replicate lines were established. These were S, the stabilisation lines, in which males with four bristles were used as fathers; $\mathrm{L}$, three lines selected for low bristle number; $\mathrm{N}$, three lines in which I hoped to observe any effects of natural selection; and $\mathrm{C}$, three control lines in which neither artificial nor natural selection would operate.

The experimental lines $\mathrm{S}, \mathrm{L}$ and $\mathrm{N}$ were set up and maintained by mating M-5 males with heterozygous females. In the control lines, wildtype males were used, and M-5 males from the progeny were discarded after being scored for bristle number. Thus, in the control lines the male M-5 phenotype was never exposed to the modifying influence of natural selection. In the $\mathrm{S}, \mathrm{L}$ and $\mathrm{C}$ lines, 7 males and 20 females were used in each generation. In the $\mathrm{N}$ lines, 10 females and 40 males chosen at random were used. This could allow for the possibility that natural selection might operate through greater mating success of four-bristled flies. With these numbers of parents, all 12 lines would be expected to show the same degree of genetic drift in allele frequency. This is discussed more fully below.

The flies were raised in $2.5 \mathrm{~cm}$ diameter vials with $14 \mathrm{~g}$ of food for the first three generations, after which $250 \mathrm{ml}$ glass jars with $56 \mathrm{~g}$ of food were used to avoid overcrowding. Food was four parts of "instant mashed potato" added to 10 parts of water (Frankham, 1973). A few pellets of dried yeast were also supplied. All cultures were raised at $25^{\circ} \mathrm{C}$. Selection was continued for a total of 17 generations.

\section{(iii) Electrophoretic analysis}

Individual flies (for $A d h$ and $E s t-6$ ) or larvae (for $A p h$ ) were homogenised in a drop of buffer and the homogenates were applied to a starch gel by means of filter-paper inserts. Thirty-six flies were scored from each line. The gel buffer was 0.076 Molar Tris- $\mathrm{HCl}, p \mathrm{H} \mathrm{8.5}$, containing $10^{-3} \mathrm{Molar}$ $\mathrm{MgCl}_{2}$, and the electrode vessels contained $0.15 \mathrm{Molar}$ phosphate buffer, $p H$ 8.8. The substitution of phosphate for the more usual borate reduced the extent of "streaking" of the samples. Esterase- 6 was stained using a mixture of $\alpha$ - and $\beta$-naphthyl acetate with Fast Blue B, alkaline phosphate with $\alpha$-naphthyl phosphate and Fast Blue RR, and alcohol dehydrogenase with isopropanol in an MTT-couped reaction.

\section{(iv) Computer simulation}

Since two of the loci are linked, and since mating is not wholly random (females mate only once), the usual theoretical model could not be used to 
calculate the variance effective number. Therefore the predicted behaviour of the lines in the absence of selection was simulated by a computer model. Recombination was simulated by a random walk method (Fraser and Burnel, 1970), using a recombination frequency between Est- 6 and Aph of 9.5 per cent. No recombination was allowed in males. Females were allowed to mate once randomly with chosen males. The random number generator was supplied with the software of the computer, a CDC Cyber 72 in the computer laboratory of Leicester University. The computer simulation was also used to establish the variance effective number of the populations.

\section{Results}

\section{(i) Response to selection}

Bristle number in the $\mathrm{C}$ lines remained fairly constant throughout the experiment. Most fluctuations were repeated in all three lines. This suggests general environmental effects, which extended to the other lines as well. The $\mathrm{L}$ lines responded rapidly to directional selection, reaching an overall mean of 0.91 bristles, showing that genes affecting bristle number are segregating despite the limited genetic variability of the founder population. The $\mathrm{N}$ lines showed little change; if anything, the mean bristle number in these lines had decreased. This result differs from that observed in the stocks mentioned above. The main difference in culture conditions was that the flies in this experiment were raised in uncrowded conditions, while the lethal-bearing stocks were often very crowded in small vials. It seems that in the less stringent environment males in all bristle number classes are equally fit, and natural selection has had no discernible effect.

The means of the $\mathrm{S}$ lines approached a value of 4 , and can be examined in terms of the distributions of bristle number. Fig. 1 shows these distributions at the 17 th generation for the $S$ and $C$ lines. These show that in at least two cases selection for flies with exactly four bristles has been highly successful.

\section{(ii) Allele frequency changes}

Table 1 gives the final allele frequencies for each of the three loci in all 12 lines. Two questions can be asked of these results. First, do the separate loci show patterns of change which are independent of the selection regimes, and second, does a particular selection regime have effects which apply equally to all three loci? Table 1 gives the means and variances of the allele frequencies obtained for two different groupings; first by taking together all allele frequencics for each locus in turn, and second, by taking together the three $A d h$ frequencics, the three $E s t-6$ frequencies and the three Aph frequencies for each set of lines in turn. Fig. 2 presents these final gene frequencies in diagrammatic form. Two features of this diagram can be seen. First, the frequency of the fast $A p h$ allele has never fallen much below the starting value of $0 \cdot 5$, and in the $\mathrm{C}$ and $\mathrm{L}$ lines it has shown a marked increase. Second, the allele frequencies show little divergence from the starting value in the $\mathrm{S}$ lines, and have diverged considerably in the other three sets of lines. 
Control
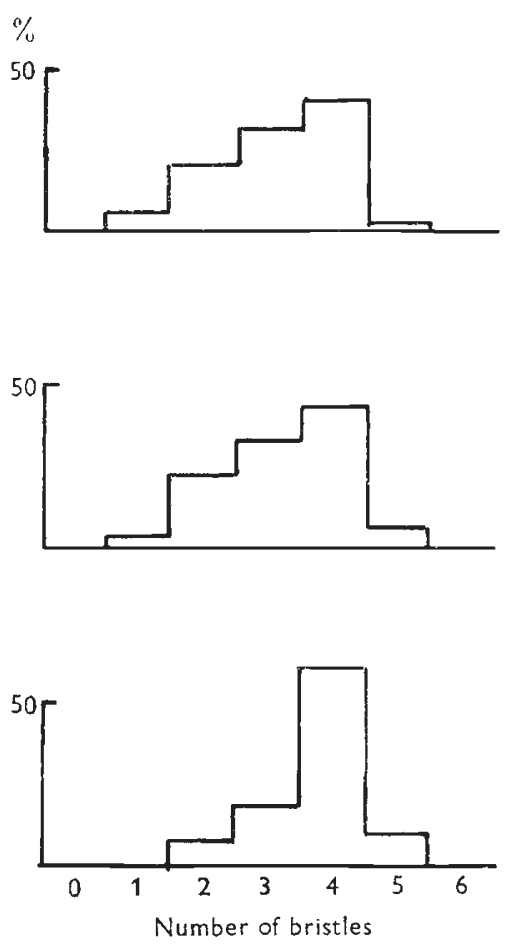

Stabilisation
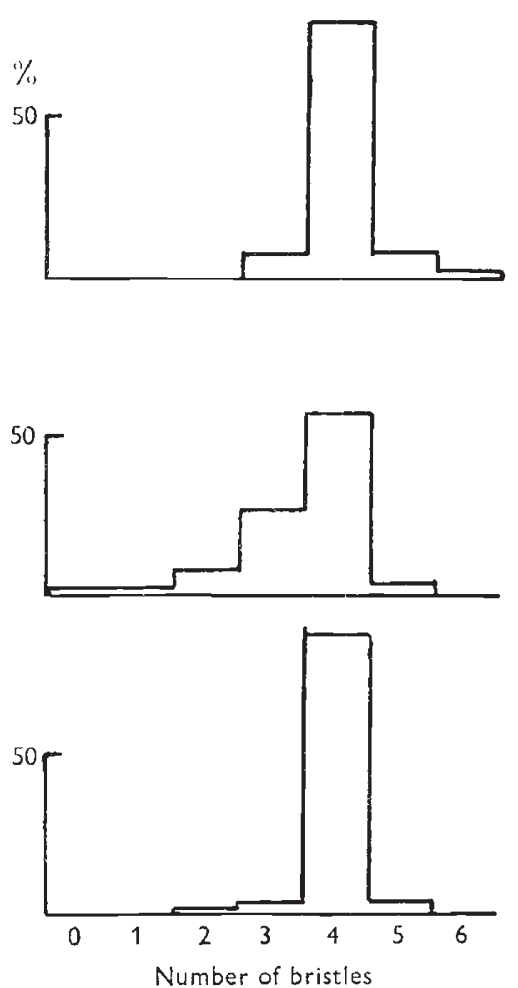

FIG. 1.-Histograms showing the distribution of bristle counts in the control and stabilisation lines at generation 17. In each case, the first, second and third lines are given in order from top to bottom.

\section{TAble 1}

Frequencies of the fast allele for each enzyme locus in all 12 lines, at generation 17 . The column headed "mean and variance" gives means and variances of the nine frequencies in the adjacent block. The bottom rows give means and variances for the 12 frequencies in each column. The actual variance, rather than the sample estimate, is given in each case.

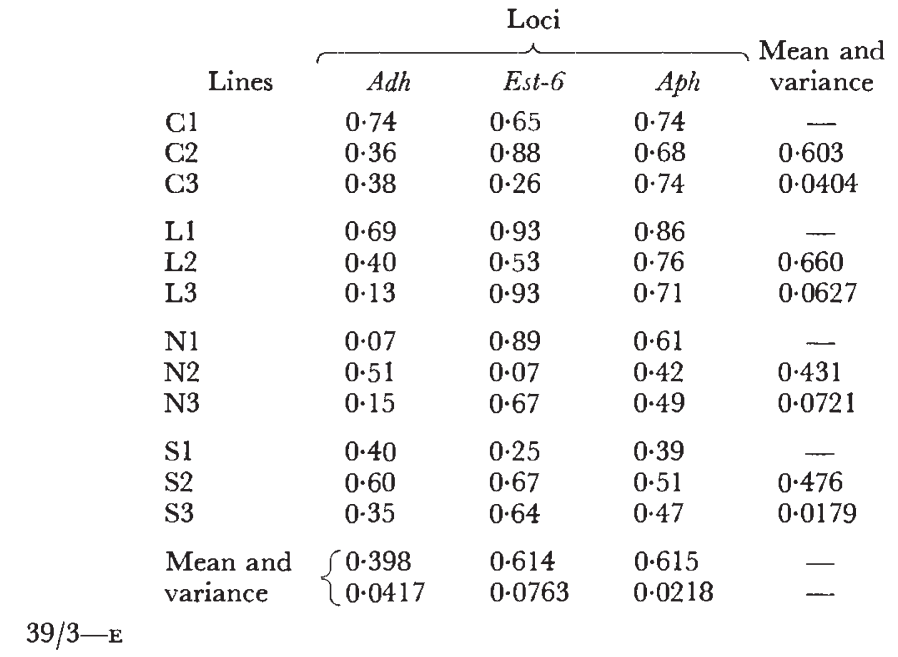




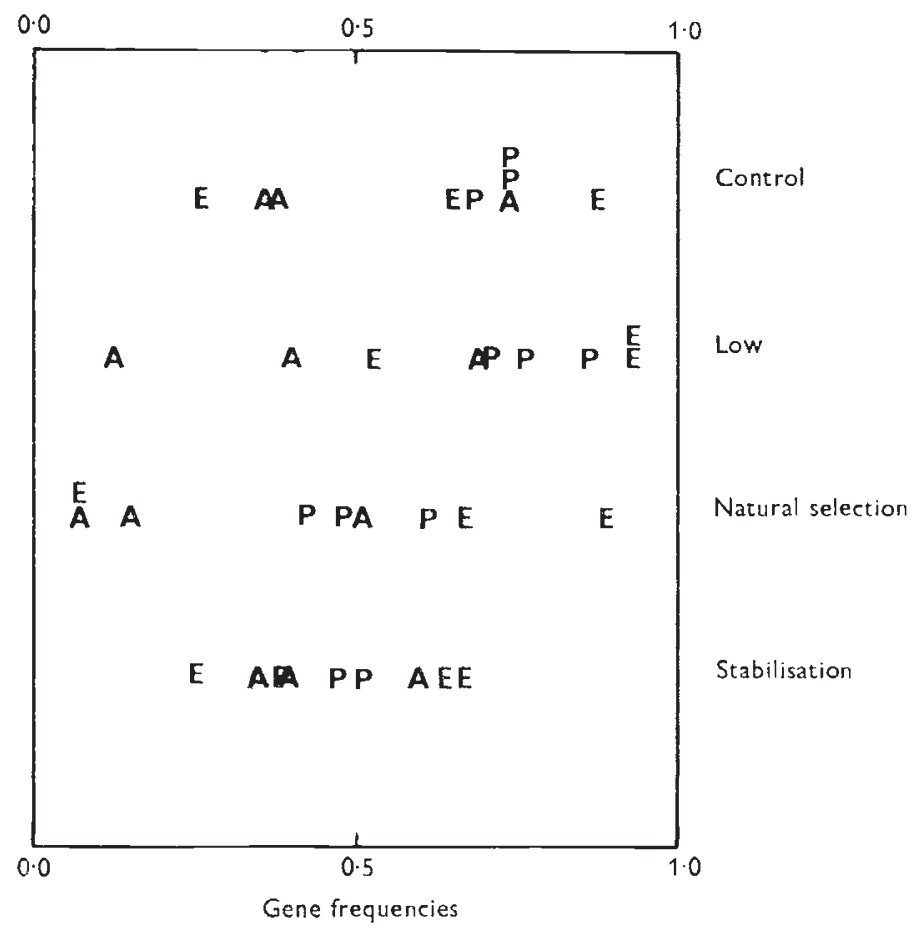

FIG. 2.-Distribution of gene frequencies at generation 17. For each of a total of 36 frequencies, the frequency of the fast allele is shown by the position of a letter on a horizontal axis. A, represents Adh; E, Est-6; and P, Aph.

\section{Discussion}

(i) Effective population size

Although the inbreeding effective population size is not affected by the mating behaviour of Drosophila females, the variance effective size, on which the sampling variancc of allele frequency depends, is affected. The computer simulation shows that the variance effective size is the same for both the $\mathrm{N}$ lines and the $\mathrm{S}, \mathrm{C}$ and $\mathrm{L}$ lines. By simulating dispersion of allele frequencies for a large number of unlinked loci, this size can be shown to be between 19 and 19.5. (The standard formula would give 20.7 for the S, C and L lines and 32 for the $\mathrm{N}$ lines.) In the analysis of changes in gene frequency at specific loci, we can therefore rcgard samples from all 12 lines as coming from a partent population with constant mean and variance of allele frequency.

\section{(ii) Aph frequencies}

The overall mcan frequency of the fast allele is $0 \cdot 615$ and the difference between this value and 0.5 gives a value of 2.58 for Student's $t$, which is significant at the 5 per cent level. This behaviour of the Aph locus is not surprising. It is analogous to the results obtained by Yarbrough and Kojima (1967) and also by Jones and Yamazaki (1974) in their strains produced from 
two homozygous lines. The intensity of selection involved is however notable. It is sufficient to overcome the effects of drift in a population whose effective size is less than 20.

The Est- 6 lies at a map distance of 9.5 units from $A p h$, and although the mean allele frequency is the same, the variance is considerably greater. Moreover, there is no significant correlation between the allele frequencies at these two loci $(r=0.49)$. The loci can therefore be said to be behaving largely independently. This suggests that the selective processes which are changing the allele frequency at the $A p h$ locus act on a chromosomal region which does not include the Est- 6 locus, or at least that the Est-6 locus is near the end of such a region.

Rendel (1976) has shown that a region of the third chromosome that includes the Aph locus but not the Est-6 locus has an important influence on scutellar bristle number. Possession of the " mutant" greatly increases the number of bristles in wild-type flies, but has little effect on scute flies. It might be claimed that a similar mutant region, with an additional pronounced effect on scute flies, was carried by my M-5 stock. I do not consider this likely. The low selection lines show the most consistent increase in the frequency of $A p h-F$, the allele carried by the wild-type stock. This implies that the frequency of mutant regions was reduced in these lines, which in turn might be expected to reduce the mean bristle number. However, in the control lines a very similar change in $A p h-F$ frequency is not accompanied by a corresponding drop in mean bristle number. Furthermore, practically no wild-type flies with more than four bristles were seen throughout the experiment.

\section{(iii) The $S$ selection lines}

All allele frequencies, regardless of locus, have remained close to the starting value. The variance of the nine frequencies can be shown to be significantly smaller than the value predicted by computer simulation. A variance ratio test is inappropriate since the distribution of $F$, the variance ratio, assumes that the sample variances come from a normal distribution, and allele frequencies after several generations of drift are far from normally distributed. That some of the loci are linked can only complicate matters.

A Monte Carlo method was used to test the significance. When the computer simulation was repeated two thousand times, the mean value of the variance was 0.0814 and in 20 cases the variance was less than or equal to the experimental value of 0.0179 . The results were insensitive to small changes in the recombination frequency. A one-tail test is appropriate since the alternative to the null hypothesis is that stabilising selection will have reduced the variance of allele frequencies. The observed variance is therefore smaller than the predicted value at a significance level of 1 per cent. This level has a sampling error of its own equal to about $0 \cdot 2$ per cent, but this cannot alter the fact that the observed variance is significantly smaller than predicted. Indeed, the predicted value itself is probably too small, since artificial selection will decrease effective population size, and increase the predicted variance (Robertson, 1961). Although most males in the later generations had exactly four bristles and were thus available as parents, the intensity of selection was greater in the early generations.

For the three other sets of lines the observed variances are close to the 
predicted values. This is as expected for the $\mathrm{C}$ lines, and is consistent with the absence of any observable selection in the $\mathrm{N}$ lines, for which the predicted value, separately derived, was 0.0806 .

\section{Conclusion}

A population will evolve when it finds itself in an environment to which it is not adapted. Such a state of affairs can be produced either by changing the environment and leaving the gene pool unaltered or by leaving the environment unaltered and establishing in it a population with a novel gene pool. Although the first possibility seems more likely in nature, the second has the same end-result and is easily accomplished in the laboratory.

In the experiment reported here, the environment (Drosophila culture bottles) has remained the same, with the additional specification that, in the $\mathrm{S}$ lines, possession of four scutellar bristles is essential for survival. But a new gene pool has been produced by crossing the M-5 and wild-type stocks. In terms of natural populations, one might imagine a small number of founders from two genetically distinct populations colonising a previously unoccupied but hospitable habitat.

The phenomena observed are magnified versions of what might take place in such a natural population. In particular, a small population size means rapid genetic drift, which in the $\mathrm{S}$ lines has been successfully opposed by fairly intense selection. In a large natural population, genetic drift is slower and selection need not be so intensc in order to oppose it. The other genetic change not cxamined directly in this experiment is the progress toward linkage equilibrium. Here again the contrast is between the artificial population, in which selection may slow down the rapid decay of the initial disequilibrium between widely separated loci, and a natural population where less intense selection will interact with the more persistent disequilibrium between closely spaced loci. It does not seem unreasonable to suppose that stabilising selection could operate in natural populations to produce, if not a rigorously stable polymorphism, then at least the long periods of quasi-equilibrium predicted by Lewontin's (1964) model, during which alleles will persist in the population at intermediate (i.e. polymorphic) frequencies.

Acknowledgment.-I am grateful to Professor P. H. A. Sneath for his comments during the revision of this manuscript.

\section{REFERENGES}

FRANKham, R. 1973. Instant mashed potato as a fly food. Drosophila Information Service, $50,199$.

fraser, A., AND burnell, D. 1970. Computer Models in Genetics. McGraw-Hill, New York. JONES, J. S., AND YAMAZAKI, T. 1974. Genetic background and fitness of allozymes. Genetics, $78,1185-1189$.

I.EWONTIN, R. C. 1964. The interaction of selcction and linkage II. Optimum models. Genetics, 50, 757-782.

Lewontin, R. C. 1974. The Genetic Basis of Evolutionary Change. Columbia University Press.

RENDEL, J. M. 1976. Is there a gene regulating the scute locus on the third chromosome of Drosophila melanogasier? Genetics, $83,573-581$.

RENDFL, J. M., AND SIILLdON, B. T.. 1960. Selection for canalization of scute phenotype in Drosophila melanogaster. Aust. 7. Biol. Sci., 13, 36-47. 
ROBERTSON, A. 1961. Inbreeding in artificial selection programmes. Genet. Res., 2, 189-194. yamazaki, T. 1971. Measurement of fitness at the esterase-5 locus of Drosophila pseudoobscura. Genetics, 67, 579-603.

YARBROUGH, K., AND KojIMA, K. 1967. The mode of selection at the polymorphic esterase-6 locus in Drosophila melanogaster. Genetics, 57, 677-686. 